\title{
Hierarchy of measurement-induced Fisher information for composite states
}

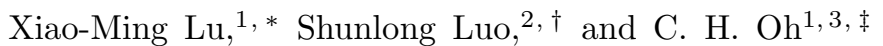 \\ ${ }^{1}$ Centre for Quantum Technologies, National University of Singapore, 3 Science Drive 2, Singapore 117543, Singapore \\ ${ }^{2}$ Academy of Mathematics and Systems Science, Chinese Academy of Science, 100190 Beijing, China \\ ${ }^{3}$ Department of Physics, National University of Singapore, 3 Science Drive 2, Singapore 117543, Singapore
}

\begin{abstract}
Quantum Fisher information, as an intrinsic quantity for quantum states, is a central concept in quantum detection and estimation. When quantum measurements are performed on quantum states, classical probability distributions arise, which in turn lead to classical Fisher information. In this article, we exploit the classical Fisher information induced by quantum measurements, and reveal a rich hierarchical structure of such measurement-induced Fisher information. We establish a general framework for the distribution and transfer of the Fisher information. In particular, we illustrate three extremal distribution types of the Fisher information: the locally owned type, the locally inaccessible type, and the fully shared type. Furthermore, we indicate the significant role played by the distribution and flow of the Fisher information in some physical problems, e.g., the non-Markovianity of open quantum processes, the environment-assisted metrology, the cloning and broadcasting, etc.
\end{abstract}

PACS numbers: 03.65.Ta, 03.67.-a

\section{INTRODUCTION}

Information is always encoded in states, often in the form of parameters. In order to read out the encoded information, one needs to distinguish different states, usually via measurements and parameter estimation. This is the fundamental issue of statistical distinguishability of states, which exhibits quite different features for the classical and quantum scenarios [1]. In the classical case, the Fisher information is the central notion in parameter estimation due to the Cramér-Rao inequality, which sets a basic lower bound to the variance of any unbiased estimator in terms of the Fisher information [2, 3]. Moreover, the maximum likelihood estimator can saturate the bound asymptotically. In the quantum realm, the information processing has a much richer structure and more potentialities which are absent in the classical world. In particular, in quantum detection and estimation, there are many (actually infinite) natural quantum extensions of the classical Fisher information, which are of relevance and significance for different tasks [4-9]. This is due to the subtle noncommutative structure of the quantum theory. Among various versions of quantum Fisher information, two prominent examples are the one based on the symmetric logarithmic derivatives [6, 7] and the Wigner-Yanase skew information [10 12].

In order to extract information from quantum states, one usually performs quantum measurements. These quantum measurements induce naturally classical probability data, and thus lead naturally to the classical Fisher information, which is deeply connected to the quantum Fisher information as revealed by the Braunstein-Caves

\footnotetext{
*Electronic address: luxiaoming@gmail.com

$\dagger$ Electronic address: luosl@amt.ac.cn

‡Electronic address: phyohch@nus.edu.sg
}

theorem [13]. More precisely, for a parameterized quantum states $\rho_{\theta}$, a celebrated version of quantum Fisher information is [, 6 , 7]

$$
F\left(\rho_{\theta}\right):=\operatorname{tr} \rho_{\theta} L_{\theta}^{2}
$$

based on the symmetric logarithmic derivative $L_{\theta}$, which in turn is Hermitian and determined by $\frac{\partial}{\partial \theta} \rho_{\theta}=\frac{1}{2}\left(L_{\theta} \rho_{\theta}+\right.$ $\left.\rho_{\theta} L_{\theta}\right)$.

If a measurement, described by a positive-operatorvalued measure (POVM) $M=\left\{M_{i} \mid M_{i} \geq 0, \sum_{i} M_{i}=\right.$ $\mathbf{1}\}$, is performed on the states $\rho_{\theta}$, then a parameterized classical probability distribution $p_{\theta}$ arises with

$$
p_{\theta}(i):=\operatorname{tr} \rho_{\theta} M_{i} .
$$

For this family of measurement-induced classical probability distributions, we have the classical Fisher information

$$
F\left(\rho_{\theta} \mid M\right):=\sum_{i} p_{\theta}(i)\left(\frac{\partial \ln p_{\theta}(i)}{\partial \theta}\right)^{2} .
$$

If a composite state $\tilde{\rho}_{\theta}$ on the tensor product Hilbert space $H \otimes K$ is an extension of $\rho_{\theta}$ in the sense that $\operatorname{tr}_{K} \tilde{\rho}_{\theta}=\rho_{\theta}$, then

$$
F\left(\tilde{\rho}_{\theta} \mid M \otimes \mathbf{1}^{K}\right)=F\left(\rho_{\theta} \mid M\right),
$$

where $\mathbf{1}^{K}$ is the identity operator on $K$ and $M \otimes \mathbf{1}^{K}=$ $\left\{M_{i} \otimes \mathbf{1}^{K}\right\}$ is a POVM on $H \otimes K$.

The elegant Braunstein-Caves theorem states that [13]

$$
F\left(\rho_{\theta}\right)=\sup _{M} F\left(\rho_{\theta} \mid M\right),
$$

where the supremum is over all POVMs. In a word, the quantum Fisher information, as defined via the symmetric logarithmic derivative, is the maximum of measurement-induced (classical) Fisher information. 
However, in practice, due to various limitations, measurements are often restricted to certain classes, such as local measurements if the system is composite. In such cases, the quantum Fisher information is usually unachievable, and the question arises as how much Fisher information can be extracted via restricted measurements. In the multi-partite system scenario, it is also relevant and important to investigate the distribution and transfer of the Fisher information over various subsystems. This leads us to the study of measurementinduced Fisher information. In this article, we will exploit measurement-induced Fisher information in order to reveal certain intrinsic structures of the underlying quantum states, with implications for practical issues.

The article is structured as follows. In Sec. II, we investigate the hierarchical structure and fundamental properties of measurement-induced Fisher information. In Sec. III, we address the issue concerning distribution of the Fisher information. In Sec. IV, we discuss transfer of the Fisher information in some practical problems. Finally, Sec. V concludes with a discussion.

\section{MEASUREMENT-INDUCED FISHER INFORMATION}

For composite systems, a technical limitation of the implementation of POVMs may naturally be induced by the tensor product structure of Hilbert spaces, e.g., for distantly separated subsystems, the local measurements are often easier than the global measurements. To investigate the distribution of the Fisher information over composite systems, we first introduce the accessible Fisher information when the POVMs are restricted. Let $\mathcal{M}$ be a set of POVMs (implying that we have technical limitation to implement all the POVMs), we define $\mathcal{M}$-induced Fisher information

$$
F\left(\rho_{\theta} \mid \mathcal{M}\right):=\sup _{M \in \mathcal{M}} F\left(\rho_{\theta} \mid M\right),
$$

where the supremum is over $M=\left\{M_{i} \mid M_{i} \geq 0, \sum_{i} M_{i}=\right.$ $\mathbf{1}\}$ in $\mathcal{M}$, and $F\left(\rho_{\theta} \mid M\right)$ is defined via Eq. (1). Obviously, if $\mathcal{M}$ is the entire set of all POVMs, then $F\left(\rho_{\theta} \mid \mathcal{M}\right)$ equals the quantum Fisher information $F\left(\rho_{\theta}\right)$ due to Eq. (3). Since the classical Fisher information is convex, it follows that the measurement-induced Fisher information is convex in the sense that for any set $\mathcal{M}$ of POVMs,

$$
F\left(\lambda \rho_{\theta}+(1-\lambda) \sigma_{\theta} \mid \mathcal{M}\right) \leq \lambda F\left(\rho_{\theta} \mid \mathcal{M}\right)+(1-\lambda) F\left(\sigma_{\theta} \mid \mathcal{M}\right) .
$$

Here $\lambda \in[0,1]$ is a real number.

We consider a bipartite system with the Hilbert space $H=H^{a} \otimes H^{b}$ and a parameterized family of bipartite states $\rho_{\theta}$ with reduced states $\rho_{\theta}^{a}:=\operatorname{tr}_{b} \rho_{\theta}$ and $\rho_{\theta}^{b}:=\operatorname{tr}_{a} \rho_{\theta}$. In this context, some natural sets of POVMs are listed in Tab. [1 see also Ref. [14]: $\mathcal{M}^{a}$ and $\mathcal{M}^{b}$ are sets of all local measurements on parties $a$ and $b$, respectively; $\mathcal{M}^{a, b}$ is the set of all joint POVMs for which $a$ and $b$ perform independently on their respective local systems; $\mathcal{M}^{a \rightarrow b}$
TABLE I: Some natural subsets of POVMs for the composite system $H^{a} \otimes H^{b}$.

\begin{tabular}{ll}
\hline \hline POVMs & Set of POVMs \\
\hline$M^{a}=\left\{M_{i}^{a} \otimes \mathbf{1}^{b}\right\}$ & $\mathcal{M}^{a}$ \\
$M^{b}=\left\{\mathbf{1}^{a} \otimes M_{i}^{b}\right\}$ & $\mathcal{M}^{b}$ \\
$M^{a, b}=\left\{M_{i}^{a} \otimes M_{j}^{b}\right\}$ & $\mathcal{M}^{a, b}$ \\
$M^{a \rightarrow b}=\left\{M_{i}^{a} \otimes M_{j}^{b \mid i}\right\}$ & $\mathcal{M}^{a \rightarrow b}$ \\
$M^{a \leftarrow b}=\left\{M_{i}^{a \mid j} \otimes M_{j}^{b}\right\}$ & $\mathcal{M}^{a \leftarrow b}$ \\
$M^{a b}=\left\{M_{i}^{a b}\right\}$ & $\mathcal{M}^{a b}$ \\
\hline \hline
\end{tabular}

is the set of joint POVMs that party $b$ performs after party $a$, conditioned on the outcomes of party $a$. Finally, $\mathcal{M}^{a b}$ is the entire set of all POVMs on the composite system.

The measurement-induced Fisher information has a natural hierarchical structure: If $\mathcal{M} \subseteq \mathcal{N}$, then

$$
F\left(\rho^{\theta} \mid \mathcal{M}\right) \leq F\left(\rho^{\theta} \mid \mathcal{N}\right) .
$$

In particular, from the inclusion relations

$$
\left(\mathcal{M}^{a} \cup \mathcal{M}^{b}\right) \subset \mathcal{M}^{a, b}=\left(\mathcal{M}^{a \rightarrow b} \cap \mathcal{M}^{a \leftarrow b}\right) \subset \mathcal{M}^{a b},
$$

we are led to the following hierarchical relations

$$
\begin{aligned}
& F\left(\rho_{\theta} \mid \mathcal{M}^{a}\right) \leq F\left(\rho_{\theta} \mid \mathcal{M}^{a, b}\right) \leq F\left(\rho_{\theta} \mid \mathcal{M}^{a \rightarrow b}\right) \leq F\left(\rho_{\theta} \mid \mathcal{M}^{a b}\right), \\
& F\left(\rho_{\theta} \mid \mathcal{M}^{b}\right) \leq F\left(\rho_{\theta} \mid \mathcal{M}^{a, b}\right) \leq F\left(\rho_{\theta} \mid \mathcal{M}^{a \leftarrow b}\right) \leq F\left(\rho_{\theta} \mid \mathcal{M}^{a b}\right)
\end{aligned}
$$

The measurement-induced Fisher information is connected to the corresponding quantum Fisher information through the Braunstein-Caves theorem and Eq. (2):

$$
\begin{aligned}
F\left(\rho_{\theta} \mid \mathcal{M}^{a b}\right) & =F\left(\rho_{\theta}\right), \\
F\left(\rho_{\theta} \mid \mathcal{M}^{a}\right) & =F\left(\rho_{\theta}^{a}\right), \\
F\left(\rho_{\theta} \mid \mathcal{M}^{b}\right) & =F\left(\rho_{\theta}^{b}\right) .
\end{aligned}
$$

Meanwhile, the measurement-induced Fisher information for the adaptive measurements can be expressed as

$$
\begin{aligned}
& F\left(\rho_{\theta} \mid \mathcal{M}^{a \rightarrow b}\right)=\max _{M^{a} \in \mathcal{M}^{a}} F\left(\rho_{\theta, M^{a}}\right), \\
& F\left(\rho_{\theta} \mid \mathcal{M}^{a \leftarrow b}\right)=\max _{M^{b} \in \mathcal{M}^{b}} F\left(\rho_{\theta, M^{b}}\right),
\end{aligned}
$$

where

$$
\rho_{\theta, M^{a}}:=\sum_{i} p_{\theta}^{a}(i)|i\rangle\langle i| \otimes \rho_{\theta}^{b \mid i}
$$

is the derived classical-quantum state induced by the local measurement $M^{a}$, and

$$
\rho_{\theta}^{b \mid i}:=\frac{\operatorname{tr}_{\mathrm{a}}\left(M_{i}^{a} \otimes \mathbf{1}^{b}\right) \rho_{\theta}}{p_{\theta}^{a}(i)}
$$

is the conditional state of party $b$ corresponding to the outcome $i$ after party $a$ performs measurement $M^{a}$, while

$$
p_{\theta}^{a}(i):=\operatorname{tr}\left(M_{i}^{a} \otimes \mathbf{1}^{b}\right) \rho_{\theta}
$$


is the probability for obtaining outcome $i, \mathbf{1}^{b}$ is the identity operator on $H^{b}$. The derived state $\rho_{\theta, M^{b}}$ is defined similarly.

To establish Eqs. (5) and (6), note that the joint probability distribution under an adaptive POVM measurement can be decomposed as

$$
\begin{aligned}
p_{\theta}(i j) & :=\operatorname{tr}\left(M_{i}^{a} \otimes M_{j}^{b \mid i}\right) \rho_{\theta} \\
& =\operatorname{tr}\left(M_{i}^{a} \otimes \mathbf{1}^{b}\right) \rho_{\theta}\left(\mathbf{1}^{a} \otimes M_{j}^{b \mid i}\right) \\
& =\operatorname{tr}\left(\operatorname{tr}_{a}\left(\left(M_{i}^{a} \otimes \mathbf{1}^{b}\right) \rho_{\theta}\right) M_{j}^{b \mid i}\right) \\
& =p_{\theta}^{a}(i) p_{\theta}^{b}(j \mid i),
\end{aligned}
$$

where $p_{\theta}^{b}(j \mid i):=\operatorname{tr} \rho_{\theta}^{b \mid i} M_{j}^{b \mid i}$ is the conditional probability of party $b$ given $a$.

Substituting $p_{\theta}(i j)=p_{\theta}^{a}(i) p_{\theta}^{b}(j \mid i)$ into the definition of $F\left(\rho_{\theta} \mid M^{a \rightarrow b}\right)$, we have

$$
\begin{aligned}
& F\left(\rho_{\theta} \mid M^{a \rightarrow b}\right) \\
= & \sum_{i j} p_{\theta}(i j)\left(\frac{\partial \ln p_{\theta}(i j)}{\partial \theta}\right)^{2} \\
= & \sum_{i} p_{\theta}^{a}(i)\left(\frac{\partial \ln p_{\theta}^{a}(i)}{\partial \theta}\right)^{2} \\
& +\sum_{i} p_{\theta}^{a}(i) \sum_{j} p_{\theta}^{b}(j \mid i)\left(\frac{\partial \ln p_{\theta}^{b}(j \mid i)}{\partial \theta}\right)^{2} \\
& +2 \sum_{i j} p_{\theta}^{a}(i) p_{\theta}^{b}(j \mid i) \frac{\partial \ln p_{\theta}^{a}(i)}{\partial \theta} \frac{\partial \ln p_{\theta}^{b}(j \mid i)}{\partial \theta} \\
= & F\left(\rho_{\theta}^{a} \mid M^{a}\right)+\sum_{i} p_{\theta}^{a}(i) F\left(\rho_{\theta}^{b \mid i} \mid M^{b \mid i}\right) \\
& +2 \sum_{i j} \frac{\partial p_{\theta}^{a}(i)}{\partial \theta} \frac{\partial p_{\theta}^{b}(j \mid i)}{\partial \theta} \\
= & F\left(\rho_{\theta}^{a} \mid M^{a}\right)+\sum_{i} p_{\theta}^{a}(i) F\left(\rho_{\theta}^{b \mid i} \mid M^{b \mid i}\right) .
\end{aligned}
$$

In the last equation, we have used the fact that $\sum_{j} \frac{\partial p_{\theta}^{b}(j \mid i)}{\partial \theta}=0$ which is implied by $\sum_{j} p_{\theta}^{b}(j \mid i)=1$.

On the other hand, the right hand side of Eq. (5) without the maximization can be calculated by the symmetric logarithmic derivative

$$
l_{\theta}=\sum_{i}|i\rangle\langle i| \otimes\left(\frac{\partial \ln p_{\theta}(i)}{\partial \theta} \mathbf{1}^{b}+L_{\theta}^{b \mid i}\right),
$$

of $\rho_{\theta, M^{a}}=\sum_{i} p_{\theta}^{a}(i)|i\rangle\langle i| \otimes \rho_{\theta}^{b \mid i}$, where $L_{\theta}^{b \mid i}$ is the symmetric logarithmic derivative of $\rho_{\theta}^{b \mid i}$. Consequently, we

$$
\begin{aligned}
F\left(\rho_{\theta, M^{a}}\right) & =\operatorname{tr}\left(l_{\theta}^{2} \rho_{\theta, M^{a}}\right) \\
& =F\left(\rho_{\theta}^{a} \mid M^{a}\right)+\sum_{i} p_{\theta}^{a}(i) F\left(\rho_{\theta}^{b \mid i}\right)
\end{aligned}
$$

Combining Eqs. (7) and (8), and making use of the Braunstein-Caves theorem for every optimization over
$M^{b \mid i}$, we obtain Eq. (5). Eq. (6) can be obtained similarly.

Measurement-induced Fisher information for any set $\mathcal{M}$ of POVMs, listed in Tab. [1, is monotonic under any local quantum operations $E^{a}$ and $E^{b}$ on $a$ and $b$, respectively, in the sense that

$$
F\left(E^{a} \otimes E^{b}\left(\rho_{\theta}\right) \mid \mathcal{M}\right) \leq F\left(\rho_{\theta} \mid \mathcal{M}\right) .
$$

To establish this, note that the classical Fisher information $F\left(\rho_{\theta} \mid M\right)$ is a functional of the probability distribution $p_{\theta}(i)=\operatorname{tr} \rho_{\theta} M_{i}$. For any quantum operation $E(X)=\sum_{\mu} E_{\mu} X E_{\mu}^{\dagger}$ with $E_{\mu}$ the Kraus operators satisfying $\sum_{\mu} E_{\mu}^{\dagger} E_{\mu}=\mathbf{1}$, we have $\operatorname{tr} E\left(\rho_{\theta}\right) M_{i}=\operatorname{tr} \rho_{\theta} E^{\dagger}\left(M_{i}\right)$, where $E^{\dagger}(X)=\sum_{\mu} E_{\mu}^{\dagger} X E_{\mu}$ is the adjoint operation of $E$. Furthermore,

$$
F\left(E\left(\rho_{\theta}\right) \mid M\right)=F\left(\rho_{\theta} \mid E^{\dagger}(M)\right),
$$

where $E^{\dagger}(M):=\left\{E^{\dagger}\left(M_{i}\right)\right\}$ is also a POVM. Therefore, for any set $\mathcal{M}$ of operations,

$$
\begin{aligned}
F\left(E\left(\rho_{\theta}\right) \mid \mathcal{M}\right) & :=\max _{M \in \mathcal{M}} F\left(E\left(\rho_{\theta}\right) \mid M\right) \\
& =\max _{M \in \mathcal{M}} F\left(\rho_{\theta} \mid E^{\dagger}(M)\right) \\
& =\max _{M \in E^{\dagger}(\mathcal{M})} F\left(\rho_{\theta} \mid M\right),
\end{aligned}
$$

where $E^{\dagger}(\mathcal{M}):=\left\{E^{\dagger}(M) \mid M \in \mathcal{M}\right\}$. In particular, for any set $\mathcal{M}$ of POVMs listed in Table I, one easily checks that for $E=E^{a} \otimes E^{b}$,

$$
E^{\dagger}(\mathcal{M}) \subseteq \mathcal{M}
$$

from which the desired inequality (9) follows.

\section{DISTRIBUTION OF FISHER INFORMATION}

In this section, we consider the distribution of measurement-induced Fisher information within bipartite systems and illustrate some extremal cases.

First, consider the relation between the locally accessible Fisher information

$$
F\left(\rho_{\theta} \mid \mathcal{M}^{a}\right)=F\left(\rho_{\theta}^{a}\right), \quad F\left(\rho_{\theta} \mid \mathcal{M}^{b}\right)=F\left(\rho_{\theta}^{b}\right),
$$

which are also called marginal Fisher information hereafter, and the global quantum Fisher information

$$
F\left(\rho_{\theta} \mid \mathcal{M}^{a b}\right)=F\left(\rho_{\theta}\right)
$$

For any product state $\rho_{\theta}=\rho_{\theta}^{a} \otimes \rho_{\theta}^{b}$, the quantum Fisher information is additive in the sense that $F\left(\rho_{\theta}\right)=$ $F\left(\rho_{\theta}^{a}\right)+F\left(\rho_{\theta}^{b}\right)$. For such cases, we say that the Fisher information of bipartite states $\rho_{\theta}$ is (exclusively) locally owned. However, for general bipartite states, due to correlations between the two parties $a$ and $b$, the quantum Fisher information can be either superadditive, i.e., 
$F\left(\rho_{\theta}\right) \geq F\left(\rho_{\theta}^{a}\right)+F\left(\rho_{\theta}^{b}\right)$, or subadditive, i.e., $F\left(\rho_{\theta}\right) \leq$ $F\left(\rho_{\theta}^{a}\right)+F\left(\rho_{\theta}^{b}\right)$. Roughly speaking, the Fisher information may be distributed exclusively in local parties, shared between the two parties, inaccessible to local parties, or in a more complex manner. The mechanism about how correlations affect the distribution of the quantum information seems unclear. In the following, we elucidate two extremal scenarios for the distribution of Fisher information: locally inaccessible and fully shared.

We say that Fisher information of bipartite states $\rho_{\theta}$ is locally inaccessible if $F\left(\rho_{\theta}^{a}\right)=F\left(\rho_{\theta}^{b}\right)=0$ while $F\left(\rho_{\theta}\right) \neq$ 0 . A simple example of this type distribution of Fisher information is $\rho_{\theta}=\left|\Psi_{\theta}\right\rangle\left\langle\Psi_{\theta}\right|$ with

$$
\left|\Psi_{\theta}\right\rangle=\frac{1}{\sqrt{2}}\left(\left|0^{a}\right\rangle \otimes\left|0^{b}\right\rangle+e^{i \theta}\left|1^{a}\right\rangle \otimes\left|1^{b}\right\rangle\right),
$$

Here, it can be easily checked that

$$
F\left(\rho_{\theta}\right)=1 / 2, \quad F\left(\rho_{\theta}^{a}\right)=F\left(\rho_{\theta}^{b}\right)=0,
$$

and for such states, the Fisher information is superadditive in the sense that

$$
F\left(\rho_{\theta}\right)>F\left(\rho_{\theta}^{a}\right)+F\left(\rho_{\theta}^{b}\right)
$$

We say that the Fisher information of $\rho_{\theta}$ is fully sharable if $F\left(\rho_{\theta}\right)=F\left(\rho_{\theta}^{a}\right)=F\left(\rho_{\theta}^{b}\right) \neq 0$. Two simple examples of such type distribution of Fisher information are as follows. The first is the classically correlated bipartite states

$$
\rho_{\theta}=\sum_{i} p_{\theta}(i)\left|i^{a}\right\rangle\left\langle i^{a}|\otimes| i^{b}\right\rangle\left\langle i^{b}\right| .
$$

Here, it can be readily checked that

$$
F\left(\rho_{\theta}\right)=F\left(\rho_{\theta}^{a}\right)=F\left(\rho_{\theta}^{b}\right)=\sum_{i} p_{\theta}(i)\left(\frac{\partial \ln p_{\theta}(i)}{\partial \theta}\right)^{2},
$$

which is exactly the classical Fisher information of the probability distribution $\left\{p_{\theta}(i)\right\}$.

The second example of the fully shared type is

$$
\Psi_{\theta}=\cos \frac{\theta}{2}\left|0^{a}\right\rangle \otimes\left|0^{b}\right\rangle+\sin \frac{\theta}{2}\left|1^{a}\right\rangle \otimes\left|1^{b}\right\rangle,
$$

for which one easily gets

$$
F\left(\rho_{\theta}\right)=F\left(\rho_{\theta}^{a}\right)=F\left(\rho_{\theta}^{b}\right)=1 .
$$

It is evident that for the above two examples, the Fisher information is subadditive in the sense that

$$
F\left(\rho_{\theta}\right)<F\left(\rho_{\theta}^{a}\right)+F\left(\rho_{\theta}^{b}\right) .
$$

\section{TRANSFER OF FISHER INFORMATION}

Based on the distribution of measurement-induced Fisher information, one may further investigate the flow of the Fisher information over composite systems. Many of the existing physical problems can be elucidated through the flow of the Fisher information, e.g., the non-Markovianity of open quantum processes [15], the environment-assisted precision measurement [16, 17], and the cloning/broadcasting of the Fisher information [18], etc. It will be interesting to investigate how measurement-induced Fisher information is transferred in such practical issues. Due to the rich hierarchy of the measurement-induced Fisher information, in general, the Fisher information will not only flow between the reduced systems, but also between the shared type and locally inaccessible type. In this section, we illustrate various features of transfer of the Fisher information though several typical examples.

Example 1. First, let us consider a scenario in which the Fisher information is transferred from the (exclusively) locally owned type into the shared type. We assume that the initial Fisher information about the parameter $\theta$ is only locally owned by party $a$, and the parameterized family of bipartite states is assumed as product states $\sigma_{\theta}^{a} \otimes \sigma^{b}$. After a unitary transformation $U$ on the bipartite system, the output states read

$$
\rho_{\theta}=U\left(\sigma_{\theta}^{a} \otimes \sigma^{b}\right) U^{\dagger} .
$$

The global Fisher information remains unchanged under the unitary transformation. If we further assume the spectral decomposition form

$$
\sigma_{\theta}^{a}=\sum_{i} p_{\theta}(i)\left|i^{a}\right\rangle\left\langle i^{a}\right|,
$$

and that $U$ has a conditional form as

$$
U=\sum_{i}\left|i^{a}\right\rangle\left\langle i^{a}\right| \otimes U_{i}^{b}
$$

then the two reduced states of the composite system read as

$$
\rho_{\theta}^{a}=\sigma_{\theta}^{a} \quad \text { and } \quad \rho_{\theta}^{b}=\sum_{i} p_{\theta}(i) U_{i}^{b} \sigma^{b} U_{i}^{b \dagger},
$$

from which we see that the reduced states of party $a$ remain unchanged, and thus the marginal Fisher formation for party $a$ remains the same. However, party $b$ gains some Fisher information, because its reduced state become dependent on the parameter $\theta$. This indicates the transfer of some Fisher information from the locally owned type to the shared type. An extremal situation for this kind of Fisher information transfer is the broadcasting of the quantum Fisher information, where all of the locally owned type of Fisher information is transferred to the shared type, see Ref. [18].

Example 2. Now we consider a scenario in which the Fisher information transfers from the locally owned type to the locally inaccessible type. We choose the input states as $\sigma_{\theta}=\left|\Omega_{\theta}\right\rangle\left\langle\Omega_{\theta}\right|$, where

$$
\left|\Omega_{\theta}\right\rangle=\frac{1}{\sqrt{2}}\left(\left|0^{a}\right\rangle+e^{i \theta}\left|1^{a}\right\rangle\right) \otimes\left|0^{b}\right\rangle .
$$


After the controlled-NOT operation

$$
U=\left|0^{a}\right\rangle\left\langle 0^{a}\left|\otimes \mathbf{1}^{b}+\right| 1^{a}\right\rangle\left\langle 1^{a}\right| \otimes\left(\left|0^{b}\right\rangle\left\langle 1^{b}|+| 1^{b}\right\rangle\left\langle 0^{b}\right|\right),
$$

the output states read $\rho_{\theta}:=U \sigma_{\theta} U^{\dagger}=\left|\Psi_{\theta}\right\rangle\left\langle\Psi_{\theta}\right|$ with

$$
\left|\Psi_{\theta}\right\rangle=\frac{1}{\sqrt{2}}\left(\left|0^{a}\right\rangle \otimes\left|0^{b}\right\rangle+e^{i \theta}\left|1^{a}\right\rangle \otimes\left|1^{b}\right\rangle\right) .
$$

For the input state, we have $F\left(\sigma_{\theta}^{a}\right)=F\left(\sigma_{\theta}\right)=1$ and $F\left(\sigma_{\theta}^{b}\right)=0$, which means that the Fisher information is (exclusively) locally owned by party $a$. For the output state, we have $F\left(\rho_{\theta}\right)=1$ and $F\left(\rho_{\theta}^{a}\right)=F\left(\rho_{\theta}^{b}\right)=0$, and thus the Fisher information is locally inaccessible to both parties $a$ and $b$.

Example 3. Finally, we consider a situation for the transfer of the Fisher information from the locally inaccessible type to the locally accessible type. This kind of Fisher information transfer is strongly related to some practical proposals on enhancing the sensitivity of precision measurement with the help of ancillary systems [16, 17]. As a simple illustration, consider the following input states $\sigma_{\theta}=\left|\Omega_{\theta}\right\rangle\left\langle\Omega_{\theta}\right|$ with

$$
\left|\Omega_{\theta}\right\rangle=\frac{1}{\sqrt{2}}\left(\left|0^{a}\right\rangle \otimes\left|0^{b}\right\rangle+e^{i \theta}\left|1^{a}\right\rangle \otimes\left|1^{b}\right\rangle\right) .
$$

Such states often emerge in the field of quantum metrology as the optimal states for high-precision measurements [19]. After the controlled-NOT operation $U$ given by Eq. (10), the output states read $\rho_{\theta}:=U \sigma_{\theta} U^{\dagger}=$ $\left|\Psi_{\theta}\right\rangle\left\langle\Psi_{\theta}\right|$ with

$$
\left|\Psi_{\theta}\right\rangle=\frac{1}{\sqrt{2}}\left(\left|0^{a}\right\rangle+e^{i \theta}\left|1^{a}\right\rangle\right) \otimes\left|0^{b}\right\rangle .
$$

Actually, this is the reversal of the process in Example 2. One can easily check that for the input states the Fisher information is inaccessible to both parties $a$ and $b$, meanwhile in the output states the Fisher information becomes accessible to party $a$. This kind of Fisher information transfer may be called the concentration of Fisher information, compared with the broadcasting of Fisher information. The concentration of Fisher information from the composite system into the measurable subsystems may help to improve measurement precision.

\section{DISCUSSION}

Though quantum Fisher information is intrinsic to quantum states, it may not be fully extractable if quan- tum measurements are restricted, which often occurs in practice. Thus it is of basic importance to study how much Fisher information can be derived via certain class of quantum measurements. This issue concerning measurement-induced Fisher information is briefly investigated here. Based on the distribution and hierarchical structure of measurement-induced Fisher information, the flow of Fisher information over composite systems is further investigated. Many physical problems can be elucidated through the flow of Fisher information, such as the non-Markovianity of open quantum dynamics, the environment-assisted precision measurement, and the cloning and broadcasting of parameter information via quantum channels.

We point out that measurement-induced Fisher information provides a convenient and meaningful tool for quantifying correlations and nonlocality, and that an initial approach for using the Wigner-Yanase skew information (a version of quantum Fisher information) in quantifying correlations is already put forward in [20]. The hierarchical structure of measurement-induced Fisher information sheds alternative lights into correlations and may be exploited for the purpose of revealing correlations from the perspective different from the conventional entropic approach.

We have only considered a single parameter case. From both theoretical and practical points of view, it will also be interesting and useful to study the multi-parameter case, which will certainly exhibit a more complex structure for measurement-induced Fisher information.

\section{Acknowledgments}

X.-M. Lu thanks X. Wang for helpful discussions. This work was supported by National Research Foundation and Ministry of Education, Singapore, Grant No. WBS: R-710-000-008-271, and by the Science Fund for Creative Research Groups, National Natural Science Foundation of China, Grant No. 10721101, the National Center for Mathematics and Interdisciplinary Sciences, Chinese Academy of Sciences, Grant No. Y029152K51.
[1] C. A. Fuchs, e-print arXiv:quant-ph/9601020 (1996).

[2] H. Cramér, Mathematical Methods of Statistics (Princeton Univ. Press, NJ, 1946).

[3] T. M. Cover and J. A. Thomas, Elements of Information
Theory (Wiley, Second Edition, 2006).

[4] W. K. Wootters, Phys. Rev. D 23, 357 (1981).

[5] H. P. Yuen and M. Lax, IEEE Trans. Inform. Theory 19, 740 (1973). 
[6] C. W. Helstrom, Quantum Detection and Estimation Theory (Academic, New York, 1976).

[7] A. S. Holevo, Probabilistic and Statistical Aspects of Quantum Theory (North-Holland, Amsterdam, 1982).

[8] M. G. A. Paris, Int. J. Quant. Inf. 7, 125 (2009).

[9] D. Petz and C. Ghinea, e-print arXiv:1008.2417 (2010).

[10] E. P. Wigner and M. M. Yanase, Proc. Natl. Acad. Sci. USA 49, 910 (1963).

[11] S. Luo, Proc. Amer. Math. Soc. 132, 885 (2004).

[12] S. Luo, Phys. Rev. A 72, 042110 (2005).

[13] S. L. Braunstein and C. M. Caves, Phys. Rev. Lett. 72, 3439 (1994).

[14] M. Hayashi, Quantum Information (Springer-Verlag, Berlin, 2006).

[15] X.-M. Lu, X. Wang, and C. P. Sun, Phys. Rev. A 82,
042103 (2010).

[16] G. Goldstein, P. Cappellaro, J. R. Maze, J. S. Hodges, L. Jiang, A. S. Sørensen, and M. D. Lukin, Phys. Rev. Lett. 106, 140502 (2011).

[17] P. Cappellaro, G. Goldstein, J. S. Hodges, L. Jiang, J. R. Maze, A. S. Sørensen, and M. D. Lukin, Phys. Rev. A 85, 032336 (2012).

[18] X.-M. Lu, Z. Sun, X. Wang, S. Luo, and C. H. Oh, e-print arXiv:1206.2821 (2012).

[19] V. Giovannetti, S. Lloyd, L. Maccone, Phys. Rev. Lett. 96, 010401 (2006).

[20] S. Luo, S. Fu, and C. H. Oh, Phys. Rev. A 85, 032117 (2012). 